report higher stress levels than other nursing disciplines (Whitebird, Asche, Thompson et al., 2013; Hospice UK, 2015.

Aim(s) The aim was to perform a comprehensive review of the literature and to analyse research on stress, compassion fatigue and/or burnout for hospice nurses, and to identify coping mechanisms that nurses and organisations can undertake. Recommendations for changes to practice will be identified and shared in this literature review.

Methods A wide-ranging search of recent literature correlated to stress, compassion fatigue and/or burnout was undertaken to encapsulate the research published over the last eleven years.

Results Nine studies were included in this literature review. The majority of the nine studies highlight that hospice nurses do not suffer with more stress than other nursing disciplines. The recent studies offer an insight into the coping mechanisms employed to avoid stress and compassion fatigue and/or burnout (Hospice UK, 2015; Montross-Thomas, Scheiber, Meier et al., 2016).

Conclusions Hospice nurses do suffer from stress and are at risk of compassion fatigue and/or burnout. The research has shown that if emotional and physical self-care and organisational strategies are utilised this can reduce the risk of compassion fatigue and/or burnout. If nurses report that these approaches are successful, further research should be undertaken to evidence the benefits of stress reducing strategies.

How innovative or of interest to hospice and palliative care is the abstract?

Some of the stressors nurses face can be directly related to the unique nature of palliative care and dealing with death and dying (Peters, Cant, Sellick et al., 2012; Hawkins, Howard \& Oyebode, 2007). Therefore, hospice nurses should be aware of self-care strategies and support available from their organisation, as it may prevent stress, compassion fatigue and/or burnout.

\section{P-269 EXPLORING THE EXPERIENCE OF PERSONAL BEREAVEMENT FOR NURSES WORKING IN A PALLIATIVE CARE SETTING}

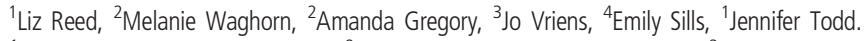
${ }^{1}$ Princess Alice Hospice, Esher, UK; ${ }^{2}$ St Catherine's Hospice, Crawley, UK; ${ }^{3}$ Phyllis Tuckwell Hospice, Farnham, UK; ${ }^{4}$ Woking Hospice, Woking, UK

\subsection{6/bmjspcare-2018-hospiceabs.294}

Background The impact on palliative care nurses of working in an environment that reminds them of their personal experience of bereavement may have a detrimental effect on their psychological wellbeing (Marcella-Brienza \& Mennillo, 2015). The emotional labour of nursing can be stressful, but when the internal reality of grief is at odds with the external reality of the professional, nurses are at risk of burnout (Brotheridge \& Grandey, 2002). Little is understood about the impact a personal bereavement has on palliative care nurses. Four hospices joined together to explore the experience of their bereaved nurses.

Aim To explore the experience of a personal bereavement for nurses working in a palliative care setting through the death of a significant relative or friend.

Outcome Make recommendations for ways hospices support nurses working in palliative care before and after the death of a significant relative or friend.
Methods Using a grounded theory approach, 13 nurses were interviewed using a semi-structured approach.

Results Initial findings suggest that if support and time to grieve is not given at the time of the death, nurses may experience a period of disintegration at a later date. Those experiencing a sudden bereavement may have different needs to those experiencing an anticipated bereavement. Grief and bereavement is a continuum experienced over a prolonged time. Anniversaries, times of stress or resonance with patient diagnosis or family reaction can trigger an emotional response challenging nurses' ability to integrate bereavement' into their lives and work. Separating the personal from the professional can take time.

Conclusion Nurses grieve in different ways and managers and employers need to allow flexibility and time for each person to grieve recognising that bereavement continues a long way past the statutory compassionate leave allowance. Recommendations will be shared with Hospice UK to consider a national initiative.

\section{P-270 WELLBEING, THE LONE HR PRACTITIONER, AND MANAGING VOLUNTEERS: CARING FOR THE CARERS' CARERS}

${ }^{1}$ Julie Davies, ${ }^{2}$ Alex Kevill, ${ }^{1}$ Dinuka Herath. ${ }^{1}$ Huddersfield Business School, Huddersfield, UK; ${ }^{2}$ Leeds University Business School, Leeds, UK

10.1136/bmispcare-2018-hospiceabs.295

In this paper we consider the role of the HR manager in managing their own self-care (Malloy, 2013) while working with hospice volunteers (Alfes, Antunes, Shantz et al., 2017). Carvalho \& Sampaio (2017) argue that there has been a lack of a strategic approach to challenges associated with the human resource management of volunteers. How does the HR practitioner regulate their own emotional labour and professional boundaries while being responsible for volunteers who support medical and nursing and other staff who care for patients and their families? How does the HR practitioner demonstrate their own engagement with the organisation without becoming over-committed? How can the HR professional devise policies and practices to optimise the benefits of volunteering (Thoits \& Hewitt, 2001) while mitigating the potential for over-commitment in hospice volunteers?

We explore theoretical concepts on boundary work and over-commitment in supporting wellbeing within an end of life care setting. Drawing on one-to-one qualitative interviews and insights from the ability, motivation, opportunity (AMO) framework and literature on SMEs (small and medium size enterprises), we highlight policy and practices to support individual wellbeing and performance management. Specifically, we focus on the concept of 'flow' (Csikszentmihalyi, 1997) in delineating boundaries between professional and personal identities.

We discuss practical implications for the career development and wellbeing of HR practitioners in UK hospices and the risks of over-commitment (Earnshaw-Smith, 1987). We also reflect on the impact of performance management and recovery time for both HR managers and volunteers. Initial findings suggest the importance of deliberate and regular interventions in the workplace to buffer work and personal space; outsourcing employee assistance for rapid responses to symptoms of distress and overload; frequent audits and appraisals to 